This item was submitted to Loughborough's Research Repository by the author.

Items in Figshare are protected by copyright, with all rights reserved, unless otherwise indicated.

\title{
Electromigration voiding in nanoindented, single crystal Al lines
}

PLEASE CITE THE PUBLISHED VERSION

PUBLISHER

(c) American Institute of Physics.

VERSION

VoR (Version of Record)

LICENCE

CC BY-NC-ND 4.0

REPOSITORY RECORD

Dwyer, Vincent M., and W.S. Wan Ismail. 2019. "Electromigration Voiding in Nanoindented, Single Crystal Al Lines". figshare. https://hdl.handle.net/2134/4985. 
This item was submitted to Loughborough's Institutional Repository (https://dspace.lboro.ac.uk/) by the author and is made available under the following Creative Commons Licence conditions.

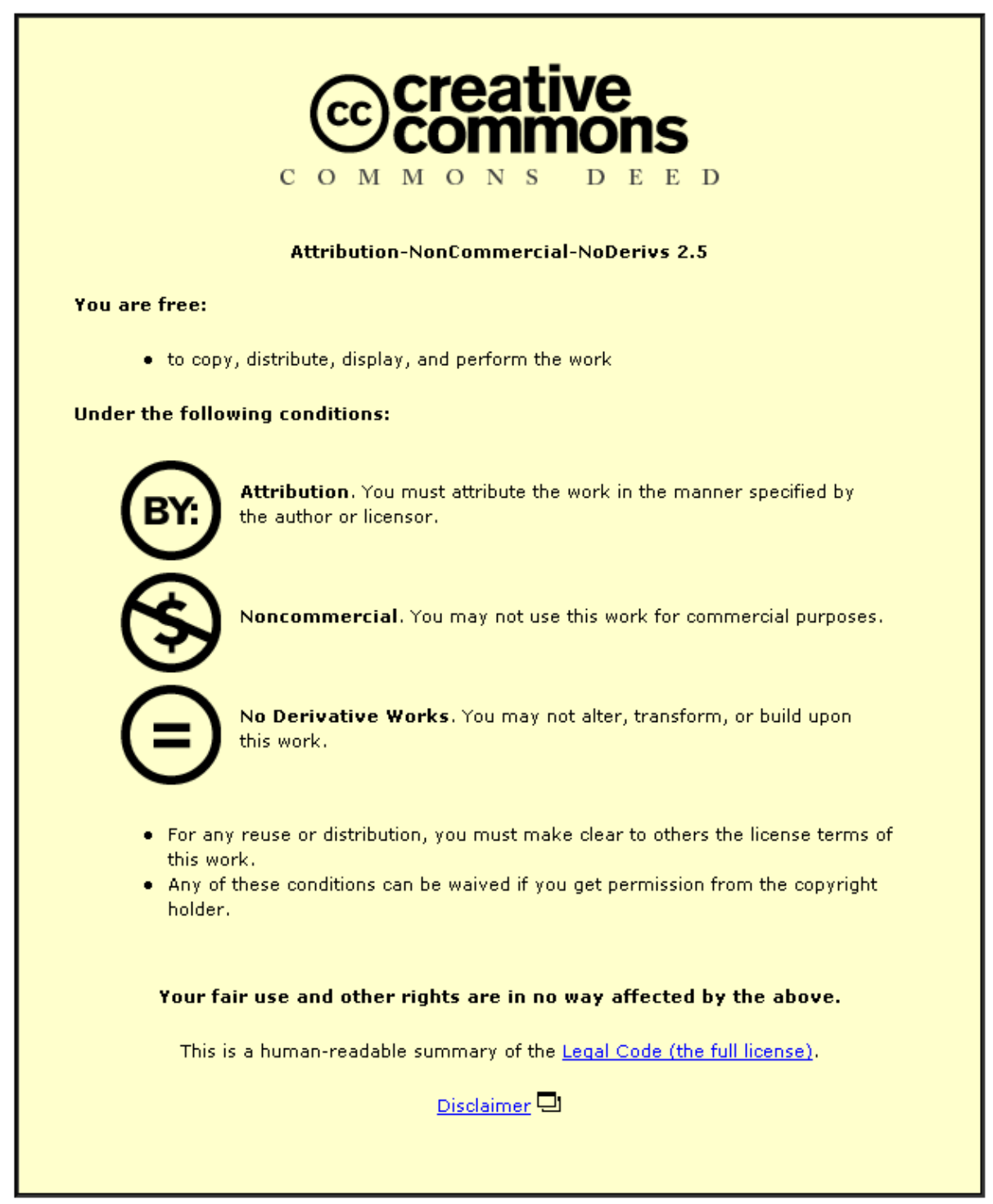

For the full text of this licence, please go to: http://creativecommons.org/licenses/by-nc-nd/2.5/ 


\title{
Electromigration voiding in nanoindented, single crystal Al lines
}

\author{
V. M. Dwyer ${ }^{\text {a) }}$ and W. S. Wan Ismail \\ Department of Electronic and Electrical Engineering, Loughborough University, Loughborough LE11 3TU, \\ United Kingdom
}

(Received 20 June 2000; accepted for publication 22 November 2000)

\begin{abstract}
We consider the interpretation of some theoretical and experimental work regarding electromigration voiding in nanoindented, single crystal aluminum lines. A recently suggested voiding criterion of a critical accumulated flux divergence is found, in fact, to be identical to the widely accepted critical stress criterion. The inclusion of the stress dependence of the atomic diffusion coefficient is shown to be vital when the steady state is characterized by $J \neq 0$, such as in the case of a void growing at a constant rate. It is found, for example, that the stress required for steady void growth, within single crystal $\mathrm{Al}$ lines, is probably significantly smaller than previously suggested. () 2001 American Institute of Physics. [DOI: 10.1063/1.1342436]
\end{abstract}

In electromigration (EM), the total atomic flux density is generally taken to be

$$
J_{A}=\frac{D_{A} C_{A}}{k T}\left(\Omega \nabla \sigma+Z^{*} q \rho j i\right),
$$

where $\sigma$ is the local tensile stress, $C_{A}$ is the atomic concentration, $D_{A}$ is the local atomic diffusion coefficient, $Z^{*}$ is the effective electromigration charge and all other variables have their usual meanings. ${ }^{1}$ Atomic diffusion occurs largely along a grain boundary network, as $D_{A}$ is significantly larger on grain boundaries than it is through the bulk. Fluxes from Eq. (1) produce relatively small changes in local vacancy concentration so that continuity may be approximated by

$$
\nabla . J_{A}+\gamma=0,
$$

where $\gamma$ is the net rate of atomic recombination at sites other than vacancies. The process is typically considered to be one of dislocation climb within grain boundaries and gives rise to an increase in local tensile stress according to

$$
d \sigma=-B \frac{d C_{A}}{C_{A}}=-\frac{B}{C_{A}} \gamma d t,
$$

where $B$ is an elastic (possibly bulk) modulus for aluminum. ${ }^{1}$ In Eq. (3) averages have been taken over the cross section of the stripe.

Assuming a Maxwell-Boltzmann population for the vacancy concentration $C_{V}$ and a hopping process for the diffusion mechanism, the atomic diffusion coefficient $D_{A}\left(=D_{V} C_{V} / C_{A}\right)$ has an exponential stress dependence, thus

$$
D_{A}(\sigma) \approx D_{A_{0}} \exp \left(\frac{\sigma \Omega}{k T}\right) \text {. }
$$

Averaging Eq. (1) over the cross section of the stripe and combining the result with Eqs. (2)-(4) yields the final onedimensional expression ${ }^{1}$

$$
\frac{\partial \sigma}{\partial t}=\frac{\partial}{\partial x}\left[\frac{B D_{A}^{\mathrm{eff}} \Omega}{k T}\left(\frac{\partial \sigma}{\partial x}+\frac{Z^{*} e \rho j}{\Omega}\right)\right]=B \Omega \frac{\partial J_{A}}{\partial x},
$$

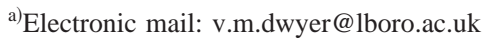

where $D_{A}^{\text {eff }}$ is the "effective" atomic diffusion coefficient caused by the averaging. For example, in polycrystalline regions $D_{A}^{\text {eff }}=\delta D_{A} / d$ for grain boundary thickness $\delta$, and average grain size $d$. Void nucleation in polycrystalline and near bamboo aluminum lines occurs largely due to the dependence of $D_{A}^{\text {eff }}$ on the position along the stripe and in typical structures this dependence is very complex. An artificial method of creating a "known" structure in single crystal aluminum lines is by nanoindentation ${ }^{2,3}$ in which fast diffusing clusters are created by mechanically damaging the crystal at certain points. We consider here some recent results on such structures.

For voiding to occur, in the standard model of electromigration described above, the tensile stress evolves until it reaches some critical value $\sigma_{\mathrm{cr}}$ at some point; void nucleation then occurs. We first consider the countersuggestion of Duan and Shen ${ }^{4}$ that nucleation occurs rather as the result of a critical accumulation (over time) of flux divergence as this is "a more feasible parameter for void formation," they claim that this gives a different nucleation point. To justify their view the authors analyze the experimental setup of Joo et $a l^{2,3}$ in which nanoindented single crystals are produced with a fast-slow-fast or similar pattern. Solving Eq. (5) they find that the two criteria give different voiding points. However, it is clear by integrating Eq. (5) that these two criteria are in fact rigorously identical, as

$$
\sigma(x, t)=B \Omega \int_{0}^{t} \frac{\partial J_{A}}{\partial x} d t .
$$

In following the work of Duan and Shen, ${ }^{4}$ we are able to reproduce their figures for stress evolution and for the atomic flux but we have not been able to reproduce their figure for the accumulated flux divergence, which we find is proportional to the tensile stress and thus is in line with Eq. (6). We can only conclude that there are numerical errors in their integration of Eq. (6).

The position of maximum stress, and thus the theoretical voiding position, depends upon the extent of the SEMinvisible plastic regions created during the indentation process. ${ }^{5}$ The nanoindented lines ${ }^{2,3}$ are created in such a 
manner that each indented segment may be considered to be continuous. This assumes that the plastic regions of each indentation overlap. As a consequence there will also exist a transition region of plastic damage between the fast and slow diffusing regions. Mechanical damage, due to indentation, appears in Eq. (5) as a position dependent diffusion coefficient $D_{A}^{\text {eff }}(x)$. Solving Eq. (1) in the steady state (with $J \neq 0$ ), for an unpassivated line, the position of maximum stress occurs at the point given by the solution to ${ }^{6}$

$$
\frac{1}{D_{A}^{\mathrm{eff}}(x)}=\frac{1}{L} \int_{0}^{L} \frac{d x}{D_{A}^{\mathrm{eff}}(x)},
$$

and $\partial D_{A}^{\text {eff }} / \partial x>0$. That is, the maximum steady state stress occurs where $D_{A}^{\text {eff }}(x)^{-1}$ is equal to its value averaged over the length $L$ of the structure. Equation (7) predicts that the site of maximum steady state stress will usually lie within this transition region and thus somewhat outside the fast diffusing region. Time-dependent stress evolution for this structure also shows that, for reasonable values of the critical stress, the voiding position will occur close to the site of maximum steady state stress. Note that, as it is $D_{A}^{\text {eff }}(x)^{-1}$ rather than $D_{A}^{\text {eff }}(x)$ that is involved in the averaging process [Eq. (7)], the position of the voiding site within the transition region will tend to lie nearer to the slow (undamaged) region than to the fast (SEM-visibly damaged) region.

For systems with a steady state corresponding to $J=0$, it is only the time to reach the steady state that is affected by $D_{A}^{\text {eff }}$. The final stress is independent of $D_{A}^{\text {eff }}$ and hence it is unimportant whether or not stress dependence is included. This is not the case for a system whose steady state (as here) is characterized by $J \neq 0$. In the initial stage, where the stress is still small, inclusion of the stress dependence makes little difference as $D_{A}^{\text {eff }}(\sigma) \approx D_{A}^{\text {eff }}(\sigma=0) \equiv D_{A_{0}}^{\text {eff }}(x)$. However as time progresses and $\sigma$ builds up the variation in $D_{A}^{\text {eff }}$ has more obvious effects.

Consider the situation of Ref. 4, but without any transitional plastic damage zone and with a spatially uniform $Z^{*}$ $=-15$. Equation (1) is solved for an unpassivated line with a slow-fast-slow structure of the same material constants as considered in Refs. 2-4 and the final stress is shown in Fig. 1(a). Note that the tensile stress gradient at the cathode end is very small. It is also true that the EM flux is much lower than the equivalent case in which the stress dependence is ignored. This is a result of the fact that compressive stress in the anode half of the interconnect reduces the effective $D_{A}^{\text {eff }}$ values there, thus reducing the overall steady state flux. Now only a small tensile stress in the cathode half of the interconnect is required to match the EM current in the anode half. Figure 1(b) shows the situation with $Z^{*}=-4$, the asymmetry is reduced but still clear. In terms of stress profile $Z^{*}$ is obviously an important, if a relatively unknown, parameter.

For this same structure (with $Z^{*}=-15$ ) we find that the maximum tensile stress in the line is $\sigma=0.314 \mathrm{GPa}$ which occurs after $\sim 1.75 \mathrm{~h}$, after which time the maximum stress drops again until it reaches the steady state value of Fig. 1(a). If void nucleation has not occurred prior to $1.75 \mathrm{~h}$ it will never occur. The maximum compressive stress in the stripe however continues to increase in magnitude to a final steady

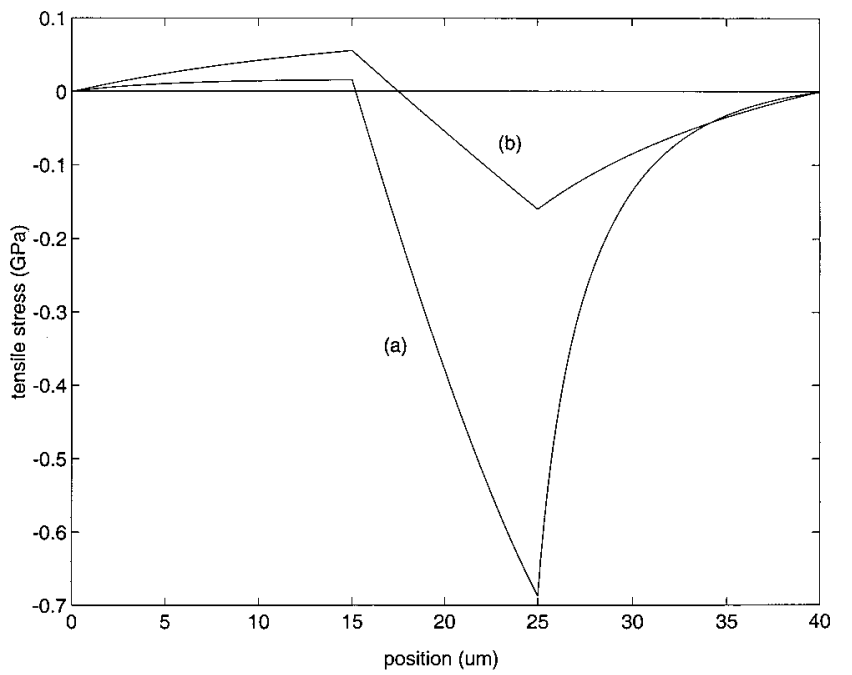

FIG. 1. Steady state tensile stress including the flux dependence of diffusion coefficient, (a) $Z^{*}=-15$, (b) $Z^{*}=-4$. Electron flow is from left- to righthand side.

state value of $\sigma=-0.688 \mathrm{GPa}$ which may be sufficient to produce a hillock-related failure. This result is in contrast to the stress-independent case in which the value of both the maximum tensile stress and the maximum compressive stress increase as $t \rightarrow \infty$.

It is clear from the discussions above that, when the steady state is characterized by $J \neq 0$, it is not valid to use steady state stress profiles (as in Ref. 2 and references therein) which do not explicitly include the exponential term in $D_{A}$, Eq. (4). The departure from the $D_{A} \approx D_{A}(\sigma=0)$ profile, indicated above, occurs quickly (well before $1.75 \mathrm{~h}$ in the example above) so that it is not generally valid to use simple linear stress profiles, obtained through ignoring stress dependence, when modeling void growth. This latter situation is considered in Ref. 2 as a model for the possible interaction between two cluster sections, each below the critical Blech length. This example is similar to the one of Ref. 4 above but considers, instead, a slow region of length $S$ separated by two fast (nanoindented) regions of lengths $L_{1}$ and $L_{2}$. Using linear steady state profiles, the authors ${ }^{2}$ find that their results to be consistent with an assumed effective charge of $Z_{f}^{*}=-15$ for the fast regions and a derived $Z_{s}^{*}$ $=-2$ for the slow region. Furthermore, because of the assumed linearity, their model only depends upon the difference in stress between the ends of the stripe $\Delta \sigma_{s s}=\sigma(x$ $=L)-\sigma(x=0)$ and not on the individual values. A value of $\Delta \sigma_{s s}=0.51 \mathrm{GPa}$ is obtained by fitting the EM flux to the observed void growth rates. Naturally if the stress dependence of $D_{A}^{\text {eff }}$ is included we obtain a rather different story as the EM current now also depends explicitly upon $\sigma(x=L)$ (through $D_{A}^{\text {eff }}$ ) but, in addition, the steady state stress profile itself is likely to be different. We set $\sigma(x=L)=0.0 \mathrm{GPa}$ here to represent stress relaxation at the void surface as in Ref. 7. In the case of the 7-S-7 structure $\left(L_{1}=7 \mu \mathrm{m}, L_{2}=7 \mu \mathrm{m}\right.$, and $S=2 \mu \mathrm{m}$ ), the stress profiles ignoring and including the $\sigma$ dependence of $D_{A}^{\text {eff }}$ are in fact very similar. Despite this there is a nearly $50 \%$ reduction in the steady state flux density $J$. This occurs as the presence of the void causes the 
stress profile to be almost completely compressive, ${ }^{7}$ thus reducing the diffusion coefficient (and mobility) through the exponential dependence on stress.

The quantitative analysis provided in Ref. 2 in order to obtain $Z_{s}^{*}$ and $\Delta \sigma_{s s}$ may also be reinterpreted including the $\sigma$ dependence. Their analysis is based on a linear fit between the segment separation $S$ and a defined variable $Y(S)$ given as $^{2}$

$$
\begin{aligned}
Y(S) & \equiv\left(\frac{J(S) k T}{D_{f}}-\frac{Z_{f}^{*} q \rho j}{\Omega}\right)\left(\frac{D_{s}}{D_{f}}\left(L_{1}+L_{2}\right)+S\right) \\
& =\frac{D_{s}}{D_{f}} \Delta \sigma_{s s}-S\left(\frac{Z_{f}^{*} q \rho j}{\Omega}-\frac{D_{s}}{D_{f}} \frac{Z_{s}^{*} q \rho j}{\Omega}\right) .
\end{aligned}
$$

The authors find a remarkable straight-line fit between $Y(S)$ and $S$, after which the right-hand side of Eq. (8) is used to obtain $Z_{s}^{*}$ and $\Delta \sigma_{s s}$. However the straight line obtained is erroneous. It occurs because $Y(S)$ so defined has an extremely weak dependence on the data $J(S)$, consequently the experimental results (i.e., the observed void growth rates) play an almost insignificant role in goodness of fit. $Y(S)$ is dominated by a linear term of slope $\left(-Z_{f}^{*} q \rho j / \Omega\right)(=9.4$ $\times 10^{13} \mathrm{~J} / \mathrm{m}^{3}$, equivalent to $C_{i}$ in the notation of Ref. 2). It is this that accounts for the quality of the fit rather than any particular trend in experimental results. To see this we need only note that, after including the experimental data in $Y(S)$, the correction to the slope is $0.124 \times 10^{13} \mathrm{~J} / \mathrm{m}^{3}\left(\equiv \alpha C_{u}\right.$ in the notation of Ref. 2) or substantially less than $2 \%$. It is better to fit the variables $Z_{s}^{*}$ and $\Delta \sigma_{s s}$ to the obtained experimental values of void growth rate against, e.g., $S^{-1}$, as shown in Fig. 2 for the 5-S-5 data. Figure 2(c) shows the best fit for stress-dependent $D_{A}^{\text {eff }}$ (this corresponds to values of $Z_{s}^{*}$ $\left.=-4, \Delta \sigma_{s s}=0.275 \mathrm{GPa}, \sigma_{L}=0.0 \mathrm{GPa}\right)$. Note that the effective EM charge in the slow region, $Z_{s}^{*}=-4$, is larger than the value of -2 suggested in Ref. 2 and perhaps in the more generally accepted range. Note also that the steady state stress across the crystal required for constant void growth rate, $\Delta \sigma_{s s}=0.275 \mathrm{GPa}$, is around half of the value of 0.51 GPa suggested in Ref. 2 and now substantially smaller than the value of $\Delta \sigma_{\text {nucl }} \approx 0.75 \mathrm{GPa}$ obtained in Ref. 2 for void nucleation. The latter value $\left(\Delta \sigma_{\text {nucl }}\right)$ is obtained from consideration of a $J=0$ steady state and is therefore independent of $D_{A}^{\text {eff }}$.

In summary, the recently proposed voiding criterion of Duan and Shen, ${ }^{4}$ in which voiding occurs at the first point to have reached a critical accumulation (over time) of flux divergence, is in fact rigorously identical to the widely accepted critical stress criterion of the standard model, Eq. (6). For nanoindented single crystal aluminum lines the theoretical position of maximum stress lies outside the indented re-

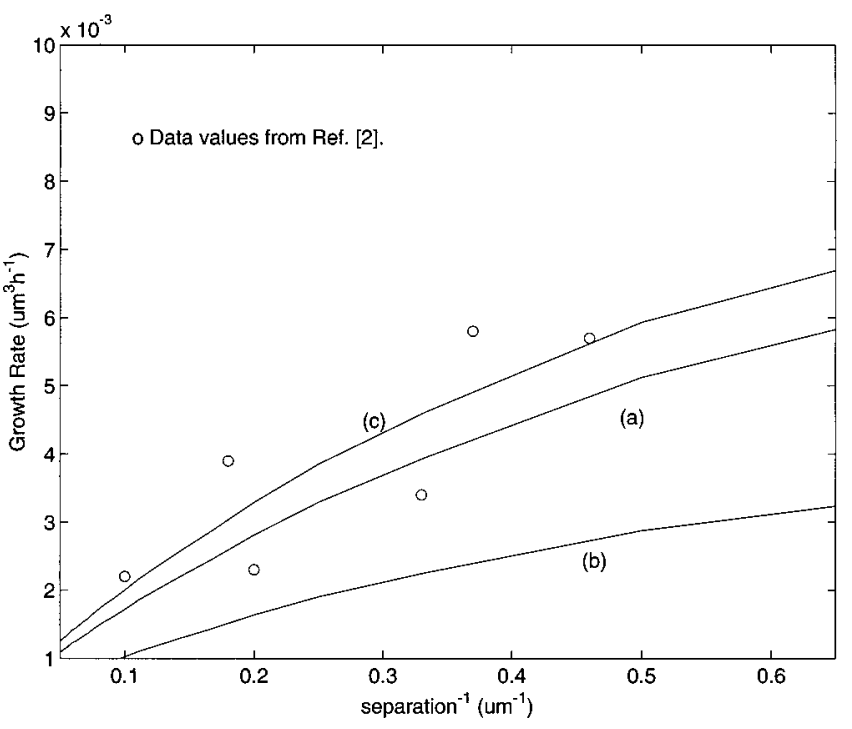

FIG. 2. Fitting of $Z_{s}^{*}$ and $\Delta \sigma_{s s}$ to the experimental results (Ref. 2) for the void growth rate $(V=\mathrm{JA} \Omega$ ) on the 5-S-5 structure. Curves correspond to (a) $Z_{s}^{*}=-2, \Delta \sigma_{s s}=0.51 \mathrm{GPa}$ ignoring stress dependence and as in Ref. 2; (b) $Z_{s}^{*}=-2, \Delta \sigma_{s s}=0.51 \mathrm{GPa}$, including stress dependence; (c) $Z_{s}^{*}=-4$, $\Delta \sigma_{s s}=0.275 \mathrm{GPa}$. Data points are taken from Ref. 2 .

gion if standard EM models are to be assumed. The distance is of the order of the range that the plastic damage extends beyond the fast region. In the analysis of systems characterized by a $J \neq 0$ steady state it is vital to include the stress dependence of the diffusion coefficient. For example, on its inclusion in the analysis of the $L_{1}-S-L_{2}$ structure $^{2}$ one finds that the stress required for steady state void growth is significantly smaller than previously suggested. ${ }^{2}$ In addition, the effective EM charge in the slow region $\left(Z_{s}^{*} \approx-4\right.$ in this example) is, perhaps, closer to a more generally accepted range. Finally, we note that $Y(S)$ given in Eq. (8) is an unsuitable parameter for fitting to the experimental data as it contains little information. The straight-line fit obtained in Ref. 2 should be disregarded.

${ }^{1}$ M. A. Korhonen, P. Børgesen, K. N. Tu, and C.-Y. Li, J. Appl. Phys. 73, 3790 (1993).

${ }^{2}$ Y.-C. Joo, C. V. Thompson, and S. P. Baker, J. Appl. Phys. 85, 2108 (1999).

${ }^{3}$ Y.-C. Joo, S. P. Baker, and E. Arzt, Acta Mater. 46, 1969 (1998).

${ }^{4}$ Q. F. Duan and Y.-L. Shen, J. Appl. Phys. 87, 4039 (2000).

${ }^{5}$ C. F. Robertson and M. C. Fivel, J. Mater. Res. 14, 2251 (1999).

${ }^{6}$ In order to separate effects we have ignored the stress dependence of $D_{A}^{\text {eff }}$ in this calculation. This is in common with other authors (Refs. 2-4). Including the dependence leads to an expression different from Eq. (7) but does not alter the conclusion that the maximum stress is not at the end of the fast region.

${ }^{7}$ M. A. Korhonen, P. Børgesen, D. D. Brown, and C.-Y. Li, J. Appl. Phys. 74, 4995 (1993). 